\title{
One If by Vaginal, Two If by C (Section): An Argument for Vaginal Delivery for Hemophilia A Carriers with Male Fetuses
}

\author{
Gregory W Kirschen ${ }^{1}$, Jennifer Pollard ${ }^{2}$ and Kimberly Herrera ${ }^{2 *}$ \\ ${ }^{1}$ Medical Scientist Training Program (MSTP), Renaissance School of Medicine at Stony Brook University, USA \\ ${ }^{2}$ Department of Obstetrics, Gynecology and Reproductive Medicine, Stony Brook University Hospital, Stony Brook, USA
}

*Corresponding author: Kimberly Herrera, Assistant Professor of Obstetrics, Gynecology, and Reproductive Medicine, Division of Maternal-Fetal Medicine, Department of Obstetrics and Gynecology, Stony Brook Medicine, Stony Brook, USA.

Received Date: November 04, 2019

Published Date: November 13, 2019

\begin{abstract}
Disorders of coagulation such as hemophilia can have profound effects on health and development beginning in the early stages of life, including pregnancy. With regard to mode of delivery planning for hemophilia A carriers bearing affected fetuses, there is little literature to guide practice and there remains little consensus regarding optimal mode of delivery. Importantly, most of the existing literature focuses solely on risk to the fetus without consideration of risk to the mother. We discuss the complex management decisions and present an argument for vaginal delivery as the "default" option, in the absence of strong maternal or fetal indications for cesarean delivery.
\end{abstract}

Keywords: Bleeding disorder; Mode of delivery; Intracranial hemorrhage; Postpartum hemorrhage

\section{Mini Review}

Optimal care for patients afflicted with disorders of blood clotting is complex and requires multidisciplinary collaboration between providers. From the birthing process forward, the hemophilia-affected fetus is susceptible to hemorrhage, with intracranial or extracranial hemorrhage of greatest concern. This brings up the question, by which mode should women carrying male fetuses potentially affected by hemophilia A be delivered? The state of the literature amounts to a relatively small number of observational studies, with no clear consensus yet emerging. Some have argued to avoid vaginal delivery, as this theoretically exposes the fetus to the greatest risk of head trauma from compression in the birth canal [1]. Further, if signs of fetal distress or dystocia develop, operative approaches such as vacuum or forceps extraction must be strictly avoided due to high risk of life-threatening iatrogenic bleeding, and morbid interventions such as emergency cesarean become necessary[1,2]. Yet, accumulating data show no significant differences in rates of severe intracranial hemorrhage or other major bleeds with either mode of delivery which is around $2-4 \%$ in either scenario, excluding operative vaginal approaches, which carry risks of $\sim 23 \%$ [3-6]. Despite this, the cesarean rate for known carriers of hemophilia A remains higher than that for unknown carriers [7], likely highlighting obstetricians' perception that cesarean delivery is safer.

We argue that the opposite is true. To date, the focus of most literature on optimal delivery mode has been on the risk of neonatal hemorrhage during the birthing process, without much discussion of other merits and risks relevant to the mother. Given that the rate of neonatal hemorrhage is virtually equivalent, other factors must be weighed in formulating a delivery plan, including risks to the mother. Our opinion is that given similar rates of neonatal bleeding, vaginal delivery is preferred in the absence of other clear obstetric indications for cesarean.

One often overlooked vulnerability of female carriers of hemophilia $\mathrm{A}$ is a bleeding diathesis in the context of major surgery. Indeed, while hemophilia A is considered an X-linked recessive disorder, there is wide phenotypic variation in terms of factor levels and bleeding diathesis, partially attributable to lionization [8]. In fact, hemophilia A carriers with factor levels of $0.4 \mathrm{IU} / \mathrm{mL}$ or below are over three times as likely to exhibit clinically significant bleeding versus controls [8]. Cesarean also carries greater risk of infection and opioid analgesia prescription compared to vaginal delivery $[9,10]$. 
Why opt for a planned cesarean then? A planned cesarean allows the multidisciplinary team of obstetricians, neonatologists, hematologists, and hemophilia nurses to congregate at the time of delivery, ensuring optimal resuscitation and seamless transitions of care that may not be possible for a patient who comes to the hospital in the middle of the night with spontaneous labor. The relationship of hemophilia A severity and risk of intrapartum hemorrhage also has not been adequately studied. For instance, severely affected fetuses may be more susceptible than mildly affected fetuses to vaginal birth-induced life-threatening hemorrhage, but this remains a theoretical risk lacking supporting evidence. In the context of hemophilia A carriers with male fetuses, providers much counsel patients using an evidence-based approach, explaining potential risks that may arise from a cesarean for which no proven neonatal or maternal benefit has yet been demonstrated. The question of vaginal versus cesarean delivery for fetuses affected by hemophilia A has no clear answer as of yet, however given the accumulating evidence of a lack of difference in terms of neonatal hemorrhage risk, we would favor a vaginal delivery unless other factors weigh heavily in support of a planned cesarean. A situation of equipoise, discussion, and informed consent are central to the decision-making process.

\section{Acknowledgement}

None.

\section{Conflict of Interest}

The authors have no competing financial interests.

\section{References}

1. James AH, Hoots $K$ (2010) The optimal mode of delivery for the haemophilia carrier expecting an affected infant is caesarean delivery. Haemophilia 16(3): 420-424.

2. Davies J, Kadir RA (2016) Mode of delivery and cranial bleeding in newborns with haemophilia: a systematic review and meta-analysis of the literature. Haemophilia 22(1): 32-38.

3. Ljung R (2010) The optimal mode of delivery for the haemophilia carrier expecting an affected infant is vaginal delivery. Haemophilia 16(3): 415419.

4. Nazir HF, Al Lawati T, Beshlawi I, AlSharidah S, Elshinawy M, et al. (2016) Mode of delivery and risk of intracranial haemorrhage in newborns with severe haemophilia A: a multicentre study in Gulf region. Haemophilia 22(3): e134-e138.

5. Andersson NG, Chalmers EA, Kenet G, Ljung R, Makipernaa A, et al. (2019) Mode of delivery in hemophilia: Vaginal delivery and cesarean section carry similar risks for intracranial hemorrhages and major bleeds. Haematologica 104(10): 2100-2106.

6. Madan B, Street AM (2010) What is the optimal mode of delivery for the haemophilia carrier expecting an affected infant-vaginal delivery or caesarean delivery? Haemophilia 16(3): 425-426.

7. Palomo Bravo A, Nunez R, Gutierrez Pimentel MJ, Nieto MD, Cos C, et al. (2016) Haemophilia neonates: mode of delivery and perinatal complications. Haemophilia 22(3): e225-e228.

8. Plug I, Mauser Bunschoten EP, Brocker Vriends AH, van Amstel HK, van der Bom JG, et al. (2006) Bleeding in carriers of hemophilia. Blood 108: 52-56.

9. Leth RA, Moller JK, Thomsen RW, Uldbjerg N, Norgaard M (2009) Risk of selected postpartum infections after cesarean section compared with vaginal birth: a five-year cohort study of 32,468 women. Acta Obstet Gynecol Scand 88(9): 976-983.

10. Badreldin N, Grobman WA, Chang KT, Yee LM (2018) Opioid prescribing patterns among postpartum women. Am J Obstet Gynecol 219(1): 103. e1-103. e8. 